\title{
Ciudad y vecinos: Cuatro ideas para la enseñanza de la vida urbana
}

\author{
Carlos Munizaga
}

\section{INTRODLCCION}

El tema de este artículo se refiere, preferentemente, a los vecinos de la ciudad. Ia lorma en que lo trate va a estar teñida por la Antropología Social que es mi campo de trabajo. Pienso que las teorías sociales están sólo a unos milímetros de la acción práctica. Por eso, siempre nuestros estudios antropológicos urbanos, se han acercado a realidades concretas como los hospitales, las escuelas, las familias en sus viviendas, las calles de una ciudad, situaciones en las que la práctica es posible.

Si aquí me refiero a materias educacionales es porque la escuela se perfila como una esperanza para que los vecinos mejoren su aprendizaje para vivir en las ciudades. Este importante papel de la escuela se incrementará a base de estudios concretos de nuestra realidad.

Al respecto, durante años estudiamos una calle de Santiago y nuestro trabajo se conoce como el proyecto de "la calle escuela". A ejemplos como éste y otros que utilizaré en esta exposición les doy la mayor importancia. Porque creo que los pedagogos están deseosos y pueden utilizar el material de investigación de la antropología urbana en su labor educativa para la vida en la ciudad.

Revisaremos aquí cuatro ideas generales que pueden ser útiles en esta tarea. Pero, especialmente, nos interesa presentar algunos ejemplos específicos con que las ilustramos. Los pedagogos podrían estructurar estos materiales, suprimir o agregar ideas. Pero, sobre todo, construir casos concretos.

Creemos que, con estas ideas, la pedagogía puede abordar cualquier aspecto relativo al medio urbano, incluyendo la ecología natural de la ciudad. Pero, creemos que, especialmente, estas cuatro ideas pueden contribuir a desarrollar un esquema mental más amplio, más flexible, que constituya una preparación 
para adelantarse a muchas de las sorpresas que el organismo urbano puede deparar en su desarrollo.

Las cuatro ideas, para partir, pueden ser las siguientes:

Primfra: Que la ciudad es un medio heterogéneo, su homogeneidad es sólo aparente. $\mathrm{Y}$ las consecuencias de esta heterogeneidad no pueden calificarse a priori como positivas o negativas si se carece de investigaciones sistemáticas, con métodos rigurosos, sobre el medio urbano.

SEgunda: Que la Ecología natural es sólo uno de los aspectos de este medio urbano. Porque otros aspectos urbanos, de igual importancia, son: el de la cultura, el de las relaciones sociales, el del mundo mental y el mundo biológico.

Tercera: Los ejemplos que, en el futuro, la educación utilice en sus programas sobre el medio ambiente urbano, deben estar ligados a situaciones concretas y críticas de la vida cotidiana y de nuestro país. Estos ejemplos deben ser enseñados en relación con disciplinas de las Ciencias Sociales y Naturales y las Humanidades. Esta idea que podríamos llamar método de enseñanza urbana, es aplicable a todas las acciones de la educación y subraya la conveniencia de un apoyo empírico, científico-interdisciplinario.

Clarta: Es el hombre el que ha construido el medio ambiente urbano. En consecuencia, hay que subrayar que el hombre es, también, capaz de intervenir en el ambiente, cambiando los aspectos urbanos naturales, culturales, sociales y mentales. Esta última idea constituye un instrumento que permitiría prevenir, para el futuro, aquellas erosiones o desbordes que provienen no sólo del ambiente natural, sino que también los que resultan del erróneo diseño material, cultural, psicológico, social, biológico de la ciudad. Esta certidumbre de que el hombre puede deshacer lo que él mismo hizo, puede superar la idea fatalista de que las catástrofes urbanas son inevitables.

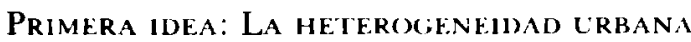

Toda ciudad, por pequeña que sea, está constituida por grupos (sean éstos personas que tienen un mismo ingreso económico o un mismo nivel educacional, o un parentesco étnico semejante). Estos grupos constituyen mundos sociales y mentales diferentes. Cualquier santiaguino observador podría hacer un mapa de mundos diferentes en la ciudad de Santiago. Y aún, descubrir mundos diferentes ubicados dentro de una pequeña Comuna. Pero, ¿cuál es la importancia de penetrar más a fondo en esta heterogeneidad de mundos? ¿Por qué la educación debería enseñar desde temprano a tener en cuenta esta diversidad?

Creo que no es posible contestar a esto si no recurrimos a unos ejemplos muy concretos de nuestro propio país. 


\section{Ejemplo de una pequeña ciudad de Chiloé}

Al estudiar una pequeña ciudad de Chiloé ${ }^{l}$, hace unos años, preguntamos a la gente si creía que el clima influía en el modo de ser y el comportamiento de las personas. Claro que la pregunta la hicimos en forma que fuera comprensible. Y, de inmediato, nos encontramos con dos mundos diferentes. Que correspondían a gente ubicada en espacios también diferentes, dentro de la ciudad. Uno era el mundo de la gente de bajos ingresos y bajo nivel cultural, que en su mayoría pensaba que el clima no tenía efectos sobre su vida mental, aunque sí sobre su físico. Otro mundo era el de la gente de mayor educación e ingresos, que creía que el clima tenía influencias en su psicología, sus depresiones y su modo de ser. Eran dos interpretaciones del mismo medio ambiente urbano en un pueblo que parecía tan uniforme, con su iglesia centenaria al centro y que podía abarcarse casi con una mirada.

Imaginemos los problemas de un planificador o de un alcalde para darse a entender entre estos vecinos, sobre temas relativos, por ejemplo, a la construcción de portales para protegerse de la temperatura, a programas de calefacción, al hablar ante una ciudad como ésta, que podríamos llamar culturalmente bilingüe.

Pero sigamos con este problema de los mundos diferentes en un medio más complejo como es Santiago, la capital de Chile. Es fácil acumular material para establecer las diferencias económicas, sociales y culturales entre las Comunas de Santiago. Aun las diferencias biológicas son fáciles de establecer: se sabe que se muere más de infartos en el sector Oriente que en el Poniente de nuestra capital ${ }^{2}$ (tasa de 49.6 en área Oriente y 24.7 en área Poniente).

Pero, falsos supuestos han llevado a pensar que esta heterogeneidad aparecería solamente cuando se comparan secciones más o menos extensas de una gran urbe, como son las Comunas.

La verdad es que la heterogeneidad cultural, mental, se descubre dentro de sectores muy restringidos de una ciudad, produciendo allí formas de vida muy particulares de cooperación, de conflicto, de traumas mentales, de dificultades de integración y organización. Estas diferencias sociales las encontramos aun dentro de las que podríamos llamar células básicas de las urbes, como son los barrios y aun las calles. Y es, sin duda, allí en el interior de estos "rincones" sociales, donde la gente vive gran parte de su verdadera vida, sentimental, intelectual, familiar, individual. Vive la vida que algunos autores llaman la "vida cotidiana".

Así, cuando estudiamos la calle Juan Vicuña, tan cerca del centro mismo de Santiago, un poco al Sur de la Av. Matta y próxima a la calle San Diego, nos

\footnotetext{
'Se trata de la ciudad de Castro, en Chiloé (IX Región). Los detalles están en Munizaga 1971,197 Ib y 1978.

${ }^{2}$ Datos suministrados informalmente por el Depto. de Estadisticas del Ministerio de Salud en 1983.
} 
encontramos con este mismo fenómeno de los mundos separados. En efecto, la gente del sector norte de la calle miraba con recelo, como a gente peligrosa e indeseable, a los de la mitad sur. Los de la mitad sur, a su vez, tenían esa misma idea negativa sobre los vecinos de la mitad norte. Sin embargo, el estudio nos reveló que tales percepciones no se basaban en hechos objetivos. Porque todos eran de bajos ingresos. Todos tenían un nivel social y cultural más o menos semejante. Pero, en el centro de la calle, atravesándola, existía un muro invisible, mucho más fuerte que el que pudiera construir cualquier arquitecto. Este "muro" dificultaba la integración de los vecinos y los obligaba a dar grandes rodeos para no transitar por la parte opuesta de la calle que constituía el mundo indeseable. Nuestro amigo, el prestigioso caricaturista Percy Eaglehurst, ha imaginado esta situación en el dibujo de la Figura 1.

Ahora, la verdad es que estas miradas de recelo ocurrían en una calle pequeña, angosta, con viviendas de un solo piso. Se podría decir que la calle tenía un diseño a escala pequeña, a escala humana. Es decir, los vecinos se detestaban a

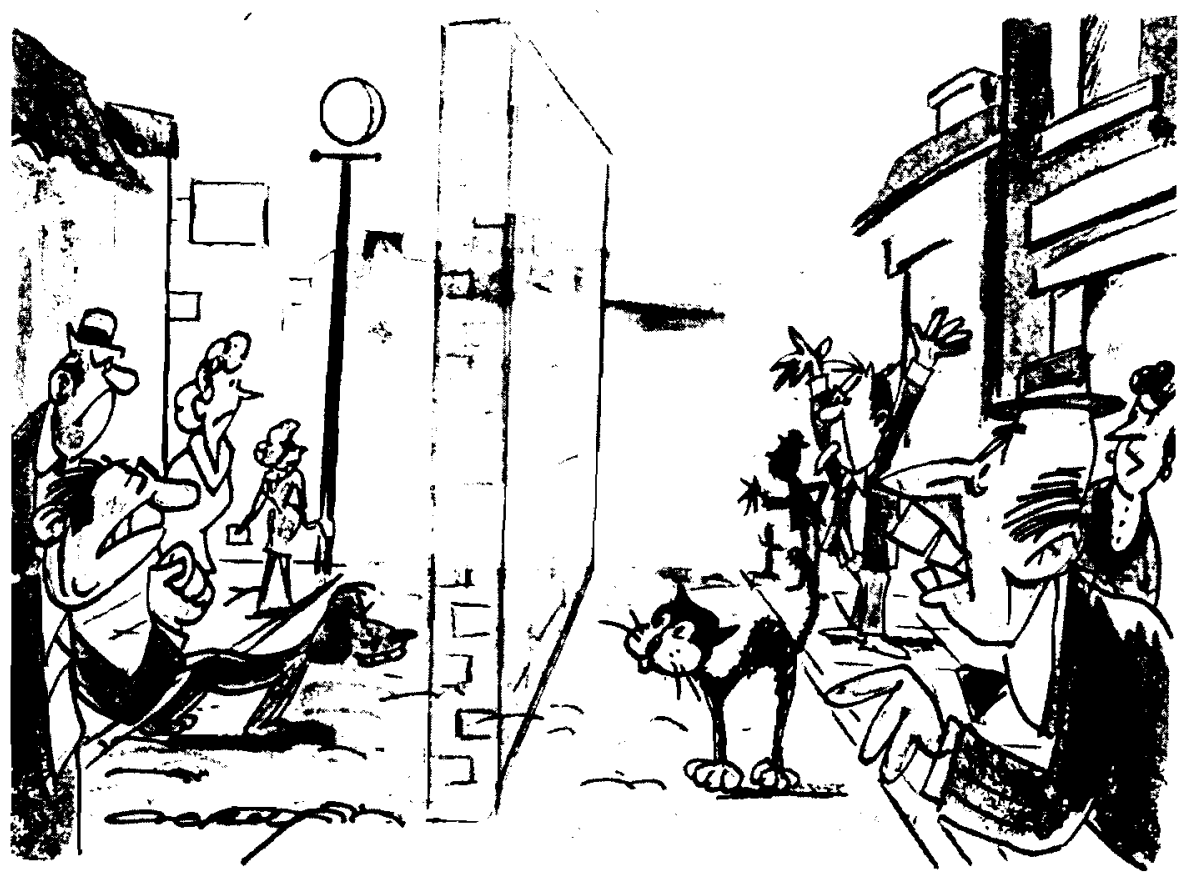

Fig. 1. Caricatura de Percy. "El Muro Invisible".

..."La gente del sector norte de la pequeña calle miraba con recelo... a los del sector sur. Y los del sector sur, tenían la misma idea de los del sector norte... existía un muro invisible que los dividí.... (frase de la conferencia dictada por Carlos Munizaga A., en la Sala Cervantes de la Biblioteca Nacional, el 23 de agosto de 1984). (Dibujo de Percy Eaglehurst, hecho especialmente para ilustrar la conferencia). 
escala humana. Y creo que lo importante, cuando se habla de escala es que, cuando no existe la escala humana, no es siquiera posible odiar, ni detestar, ni tener conflictos propiamente humanos. Pero tampoco, si no hay escala humana, se puede simpatizar, querer, saludar. $O$, tal vez, sin la "escala humana" no se puede aprender a vivir: o no nos podemos preparar para vivir dentro de escalas humanas más grandes o más abstractas. Creo que deberíamos distinguir entre escalas humanas "pequeñas" y "grandes", siendo, tal vez, las pequeñas, requisito para vivir, después, en las segundas.

Al encontrar esta pequeña unidad vecinal, de unos cuantos metros, con relaciones teñidas con tantas cargas afectivas, con tantas distorsiones de percepción, no habiamos encontrado nosotros, como se pensaría, una pústula urbana. En realidad, no habíamos hecho sino llegar a este tejido intersticial profundo, complementario, "invisible", en el que relaciones sociales que se ocultan bajo un mismo nombre, como la amistad, los saludos, la cooperación, el respeto, el visitarse, el mirarse, tienen significados y calidades completamente diferentes de los que nos enseñan los diccionarios, significado que sólo se aprehende cuando se penetra y se vive dentro de estas pequeñas células vitales.

Es importante que la educación conozca y explique la existencia de estos mundos urbanos mentales y sociales, separados por muros invisibles. Que conozca los particulares tejidos de vida con que estos mundos están constituidos y que, para entenderlos, no basta manejar los nombres de las cosas, de los sentimientos o de los valores. Esto, para que los vecinos del futuro, que son los niños de hoy, puedan transitar entre estos mundos, familiarizarse con su existencia, eludir los traumas que produce lo desconocido y aspirar a establecer entre estos mundos "particulares" los lazos necesarios para la participación y el desarrollo en una escala más amplia. Pero, sin abrigar la necia esperanza de que hay que ignorar esta diversidad de mundos o que ella decrecerá, para convertirse la urbe en un pacífico paraíso, sin conflictos. Porque ni los románticos pueblitos campesinos poseen tal felicidad, como la imaginan aquellos que van al campo a hacer pic-nics los fines de semana.

Los estudios del antropólogo Oscar Lewis, en Tepoztlán, en México, mostraron que aún en este pequeño pueblo campesino, idílico paraíso, existía un alto grado de conflicto, de tensiones, de patologías, de homosexualidad latente. Encontró que las muchachas, víctimas del control social, donde todos se conocen y vigilan, cuando lograban ellas salir de vacaciones a otros pueblos, volvían con un color más sonrosado, en lugar de la piel cetrina que les producía el ambiente psicosocial de Tepoztlán. Tepoztlán era un pueblo chico y un infierno grande.

Y la urbe es un tejido de múltiples células, cuya calidad está muy lejos de conocerse. Más aún, es posible que estén por descubrirse otros tipos de células, como las llamadas "redes", "cuasigrupos", etc., que pueden tener un papel importante para entender por qué ocurren ciertos inesperados fenómenos de participación, de conflicto, de desarrollo urbano.

Desde el punto de vista de la educación, es conveniente que pensemos que 
uno de los resullados de esta multiplicidad de mundos es una multiplicidad de diferentes percepciones de la ciudad. Al mismo tiempo, si nos ponemos en el lugar de viajeros que visitamos estos diferentes mundos dentro de nuestra ciudad, es posible que nos encontremos más satisfechos corporal, social, afectivamente, cuando penetramos en aquellos que coinciden con nuestra propia cultura, personalidad e intereses.

¿Y qué dicen los expertos sobre la ciudad del futuro en este aspecto de la diversidad? :cómo será Santiago? Cientistas sociales, como loffer, piensan que muy lejos de esperar una homogeneidad social, hemos de esperar, en el futuro, una diversificación y multiplicación mayor de los grupos en sus intereses, su cultura y sus valores. Los nedios de conmunicación de masas, la prensa y la televisión, en lugar de homogeneizar, parece que contribuirán a la multiplicación de estos grupos, de estos ojos que mirarán el mundo de la ciudad con diferentes visiones.

La Educación, pues, debe prepararse para conocer esta realidad desde temprano y para construir en los vecinos del futuro una estructura mental y de valores que les permita un lenguaje común, por encima de las diversidades. Aquel que se quede para siempre, exclusivamente, en uno de estos pequeños puntos, en uno de estos mundos de barrio, ricos de sensaciones, "ganará" su barrio o su calle, pero "perderá" el mundo urbano del hombre, en su valiosa totalidad. Por otra parte, el que pretenda "graduarse" o "doctorarse" de vecino "total" de la ciudad, sin haber "cursado" su pequeño mundo próximo se perderá en la abstracción desorientadora de la gran urbe.

Por otra parte, parece que la ciudad no es un ente metafísico que per se urbaniza, moderniza al hombre. Son elementos que están dentro de ella los que producen esta mutación mental. Y uno de estos elementos, tal vez el más decisivo para fabricar mentes urbanas modernas es la Escuela (INkE1.s. y SMITH, I974). No basta entonces estar en la ciudad, para tener la calidad de "urbano".

¿Será, entonces, el gran desafío del diseño del medio ambiente urbano y el de vivir plenamente en la ciudad, el mantener el equilibrio entre lo local y lo general, entre lo pequeño y lo grande, entre lo indizidual y lo social? ¿Y no será la escuela la agencia fundamental para lograr este equilibrio?

II

Seglnda idea: El medio lirbano comprende aspecitos naterales; SOCIALES O DE GRUPUS; CULTURALES,

(O DE IDEAS, NORMAS Y CONOCIMIENTOS); MENTALES

(RELATIVOS A LA PERSONALIDAD Y EL. PENSAMIENTO) Y ASPECTOS BIOLÓGICOS

Como resultante de esta segunda idea y de la primera, se desprende la gran complejidad dèl medio urbano. Esta complejidad puede explicar por qué, cuan- 
do la masa de los vecinos, de las autoridades, de los medios de comunicación quieren dialogar sobre la ciudad, surge un rumor ininteligible que está lleno de contradicciones, de posiciones agresivas, negligentes o excesivamente eufóricas y de percepciones erróneas de la ciudad.

En efecto, tenemos, por una parte, las percepciones diferenciales de estos mundos. Y por otra, el hecho de que estos mundos aparte tienen en su interior relaciones que varían en intensidad, varían en simpatía, en calidad, en fuerza. Vamos a encontrar que, por ejemplo, la amistad, el respeto, la autoridad, toman múltiples formas. De tal manera que resulta engañoso hablar sobre estas ideas que creemos casi inmutables porque ellas tienen, dentro de cada mundo, significados completamente diferentes.

En esta complejidad urbana, encontramos por una parte las estructuras jurídicas, administrativas de la ciudad, relativamente especificadas en códigos y en ordenanzas. Pero por otra parte, encontramos las estructuras, las conductas INVISIBLES, INTERSTICiAles. Estas últimas son grupos informales, relaciones de amistad, de parentesco, relaciones producidas por el temor, por los intereses; están los sistemas que alguien llamaría de corrupción, el "hacer la vista gorda", etc. Entre éstos, en algunos países se ubica la mafia, el cacique político, el espía industrial, el soplonaje que facilita la acción de la policía frente a la delincuencia. Estas formas urbanas invisibles, como lo han pensado los antropólogos, se adhieren, perturban, pero muchas veces mejoran la dinámica de la estructura urbana. Nacen, a veces, estas formas ocultas, en los barrios, en las industrias, en las oficinas públicas, como elementos que suplen funciones que el órgano administrativo urbano no puede todavía cumplir. Y los antropólogos están atentos a los nacimientos, a veces silenciosos, modestos, de estas formas u otras nuevas que pueden cambiar el sentido de la vida urbana, en el futuro.

Por esto, es muy difícil aseverar que una estructura urbana está tomando una dimensión inadministrable o que su administración formal debe ser erradicada. Antes de decir nada tenemos que estudiar toda la organización intersticial invisible de la ciudad, la que no siempre aparece en los tratados de "anatomía jurídica, municipal o estatal". Antes, tenemos que pensar que todavía no se han inventado todas las posibles formas sociales que pueden contribuir al armónico desarrollo de una ciudad.

Queremos poner sólo un ejemplo de un déficit urbano que parece producto de la gigantesca dimensión y diseño material de la ciudad. Este síntoma patológico que puede afectar a los vecinos, podríamos llamarlo de desorientación. En efecto, se ha dicho que algunas novelas hablan del hombre perdido en la ciudad y esto no es tan imaginativo o de ficción; porque en realidad el hombre está perdido en el laberinto urbano, y esto es efectivo no sólo si nos referimos al aspecto material de la urbe. Porque también está perdido en otros aspectos, ya que ignora, por ejemplo, muchas vías y significados que tienen los tejidos intersticiales que hemos señalado, que son de carácter grupal, mental y cultural. 
Otra vez, nuestro amigo "Percy" me ha auxiliado imaginando esta desorientación en una caricatura (Fig. 2).

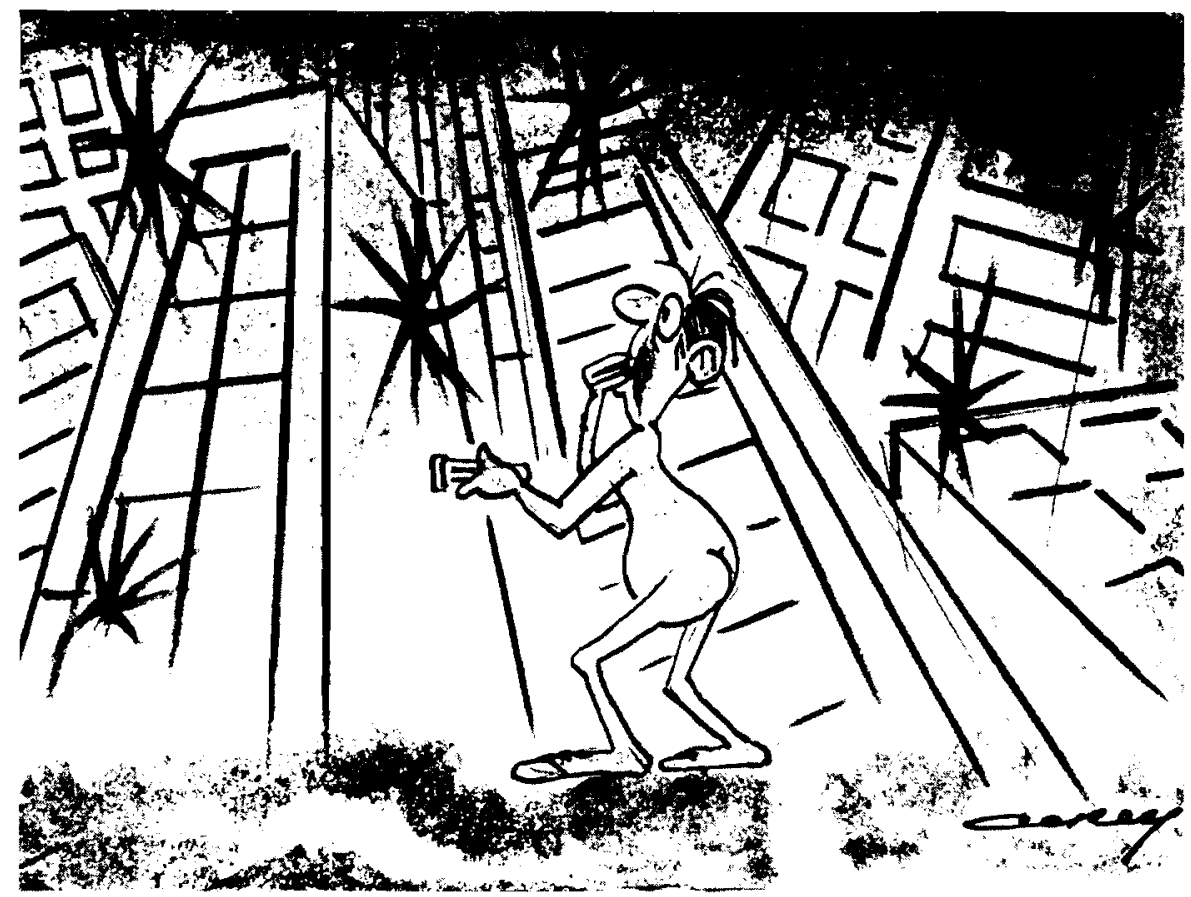

Fig. 2. Dibujo de Percy. "El hombre Perdido en la Ciudad".

Restrinjámonos, pues, a la desorientación producida por el diseño material de la gran urbe. Esta desorientación se ha señalado para lugares como Tokio y Beirut, donde se dice que es casi imposible que un individuo llegue a un lugar guiándose por las indicaciones que le suministra un vecino.

Llega un momento en que, en ciudades como Santiago, necesitamos de sistemas de coordenadas para orientarnos, o de hitos de referencia. La Psicología pedagógica señala muy bien que el uso temprano de mapas simples para orientarse, da a los individuos un instrumento que los habilita para dominar su ambiente y que, al mismo tiempo, les confiere seguridad en sí mismos. Y probablemente ayuda a desarrollar su capacidad de abstracción. La escuela debe suministrar temprano a los niños y a los adultos métodos para esta tarea de orientación física, como el uso de la brújula. Además, proponemos que una serie de lugares históricos o prehistóricos en Santiago pudieran constituir una especie de gran constelación de referencias. Por ejemplo, al Oriente, el cerro El Plomo con su famosa momia incaica; al Norte, la gran Piedra de Tacitas del Cerro Blanco, en el 
anțiguo barrio Recoleta; al Sur, la fortaleza o pucará incaico en el Cerro Chena, en San Bernardo y, entre estos puntos, algunas referencias a habitantes prehistóricos de hace un par de milenios, en la Radio Naval en la Quinta Normal (ver Fig. $3)$.

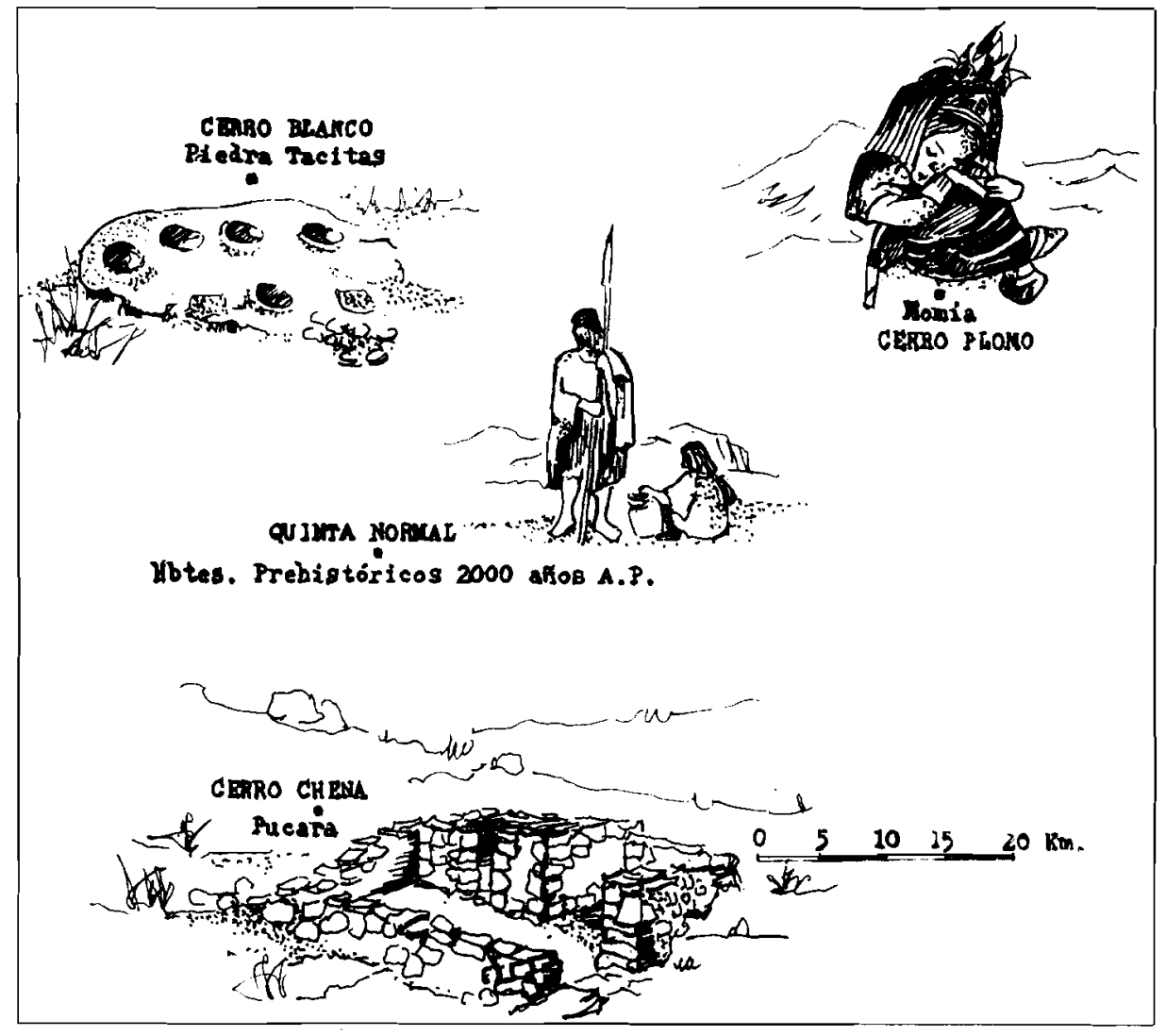

Dibujo de Miguel Bahamondes. Cartografia de Ailvona Cormeju.

Fig. 3. Hitos arqueológicos de referencia que se sugieren para orientarse en la ciudad de Santiago, capital de Chile.

Se advierte: al norte el Cerro Blanco con su gran piedra de tacitas que fue un posible adoratorio. Al oriente, el Cerro El Plomo. donde se encontró la momia incásica congelada, la que se puede ver hoy en el Museo Nacional de Historia Natural en la Quinta Normal de Santiago. Al sur. Quinta Normal, con un sitio de habitantes prehistóricos de 2.00() años ames del presente y más al sur. el pucara de Chena, una fortaleza incaica que ha sides reconstruida.

Y este gran mapa de la Figura 3 no quiere decir que no tenga en su interior una escala humana. Porque en realidad, la superficie que encierra este polígono urbano contiene multitud de unidades de pequeña escala humana. Y pensamos 
que el hombre que se ha formado en escalas pequeñas humanas está capacitado para pasar a vivir y pensar, después, sin deshumanizarse, dentro de escalas humanas "grandes", como la de la Figura 3. En realidad, la vivencia humanista, social, sentimental y científica de los pequeños mundos de la ciudad, puede permitir la elaboración de otras escalas "mayores" de orientación, basadas en hitos naturales, culturales, religiosos, estéticos. Es ésta una labor que los vecinos deben esperar de los cientistas, los artistas, los literatos, los educadores, los filósofos, los geógrafos y, también con el mismo valor, del hombre común y de la sabiduría popular.

I I I

Tercera idea: La educación urbana debe apoyarse, METODOLÓGICAMENTE, EN MATERIALES COMO, POR F]EMPIO, LOS ESTUDIOS DE. CASOS

Esta idea constituye más bien una aproximación metodológica. Sugerimos que los programas de educación relativos al medio ambiente urbano se estructuren a base de historias de casos. Que éstos deben estar basados en experiencias de la vida cotidiana. Y que su enseñanza debe vincularse a las Ciencias Sociales y Naturales, en los programas de estudio.

Creo que la temperatura vital es un buen tema para ilustrar esta idea, con casos concretos, y sirve para tomar conciencia de otros problemas de la utilización de la energía del ambiente para el desarrollo de la vida total de los individuos.

Sabemos que se requiere un óptimo de temperatura tanto para la vida de los viejos como la de los jóvenes. En algunas investigaciones que hicimos en el Sur de Chile (ver Nota 1), hace algunos años encontramos que en las viviendas, aun de gente de ingresos económicos muy bajos, se vivía fundamentalmente en la cocina-comedor en que está ubicada la cocina de hierro, también llamada "estufa". La temperatura por irradiación del hierro era de unos $22^{\circ}$ Centígrados. La gente vivía allí placenteramente reunida en la cocina, yendo a los dormitorios, mucho más fríos, sólo para dormir. Curiosamente en esos años, 1960 más o menos, la Organización Mundial de la Salud recomendaba esa temperatura como óptima para los países desarrollados. (Véase fotografías 1 y 2).

Tengo la convicción que, con motivo de ese estudio, tomé conciencia, por primera vez, de lo que es la temperatura vital, al comparar la difícil vida que en la ciudad de Santiago, incluso las familias acomodadas, tienen en invierno.

Creo que es conveniente que, en la escuela, se enseñe a los niños, desde los primeros cursos, cuáles son estas temperaturas vitales para la vida y se les enseñe a manejar los termómetros de ambiente que los encuentro que están muy olvidados. Faltan en los laboratorios de física en los liceos y, en ese tiempo, me fue muy difícil encontrar uno en el Sur para trabajar en las viviendas. 


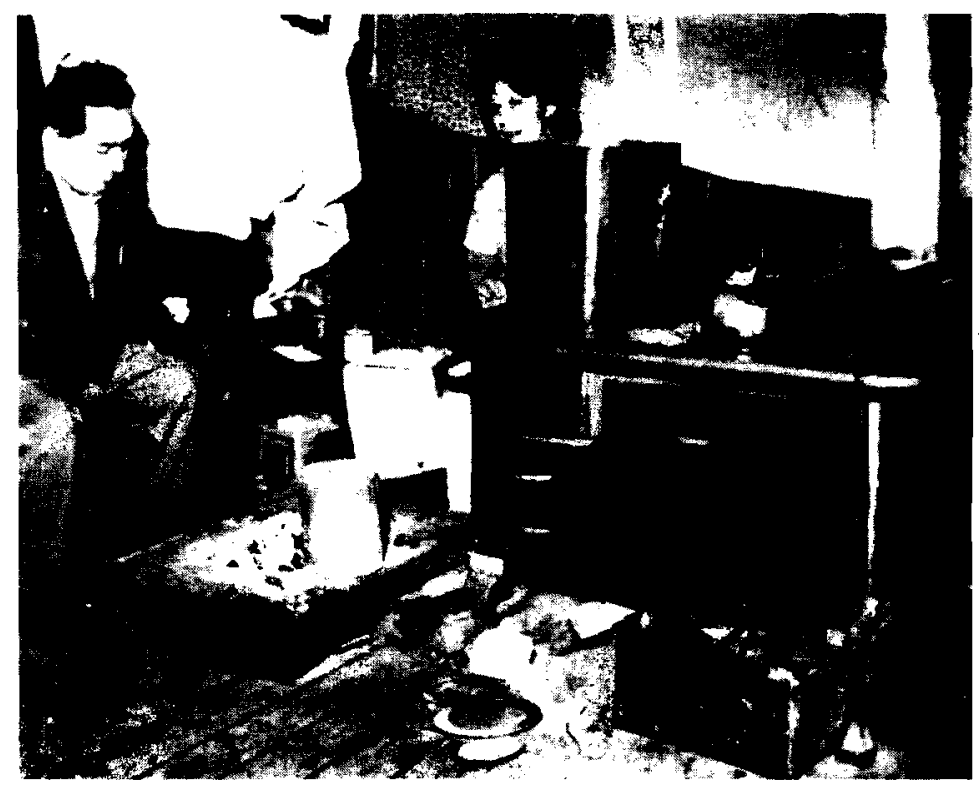

Fono 1. Interior de cocinat-comedor (estuta a leña), en loggar obremo en Castuo.

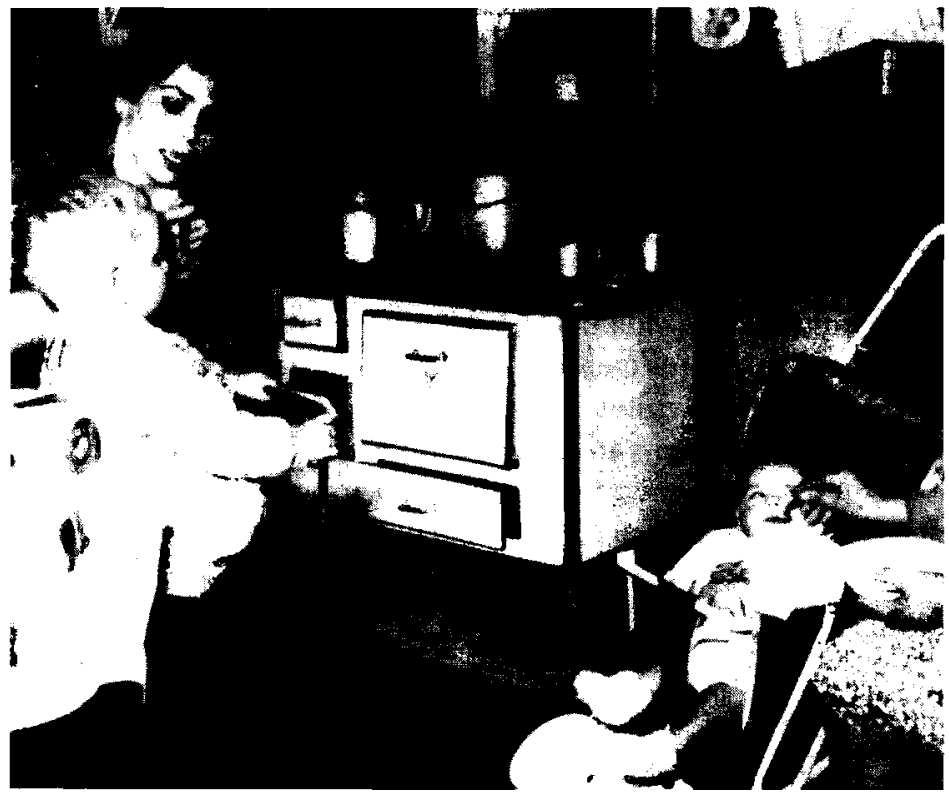

Foto 2. Interior de cocina-comedor (estuta a leña), en hogat urbano de estralocomómico altor. (i) Cisilo. 
Creo que deben utilizarse en la educación estudios de casos como los de la cocina-comedor de Chiloé, donde podemos hablar de una madre-cocina o de una madre-temperatura. Esta figura de la temperatura-madre fue para mí mucho más importante, y así lo es hasta hoy, que la temperatura que bondadosamente prodigaba a Egipto el Padre-Sol, tal como se nos enseñaba en los cursos de historia del liceo. Porque el Padre-Sol del misterioso Egipto nunca me impactó emocionalmente como la madre-cocina de Chiloé. Son éstos, temas puntuales vinculados a la discusión de los contenidos de los programas de estudio, si se quiere contribuir a la construcción de una imagen y de una "identidad" latinoamericana y chilena.

Estimo, además, que temas como los que relacionan el consumo de energía con la temperatura del ambiente, deben estar integrados en los programas de la enseñanza de las disciplinas de las ciencias sociales y naturales. A la física, la química, la biología, la antropología. Que, junto con la ley de la dilatación de los metales por el calor, se enseñen los requerimientos de energía calórica vital de nosotros, de nuestros niños, de nuestros padres y abuelos ancianos. Que se grave intensamente la necesidad de generar temperaturas vitales para una vida digna. Que en las clases de biología y de física se enseñen los requerimientos energéticos de temperatura, etc., para que la sangre circule de una manera regular y pueda irrigar un cerebro mientras piensa, por ejemplo, en la escuela. En unas escuelas de Chiloé registré temperaturas, en la sala de clases, que no alcanzaban los $10^{\circ}$. Que se enseñe la potencialidad social, moral de la temperatura: por ejemplo, la temperatura vital, puede, en pleno invierno, facilitar la interacción social del grupo familiar, o las relaciones entre el maestro y sus alumnos. Recuerdo, que hace años, no sé si es un chiste, un alto funcionario de Santiago fue a un liceo de esta capital y les preguntó a los alumnos si solicitaban algo. Los niños le dijeron que sentían frío en la sala. Y la respuesta de este alto funcionario, no sé si es un chiste o no, fue: "bueno, si tienen frío, salten".

Naturalmente que la enseñanza de tópicos como la energía y su uso para la elevación de calidad de la vida urbana debe revisarse constantemente a la luz de los nuevos descubrimientos científicos y la tecnología. En esta materia, el profesor Juan Grau, ecólogo chileno, me ha dicho que en EE.vu. se aspira hoy a óptimos más bajos que no van más allá de los $18^{\circ}$. En todo caso, es difícil si pensamos obtener en Santiago, en invierno, estos $18^{\circ}$, por los costos de la calefacción. Por eso es que, junto con enseñar de un modo in tegral la significación física, biológica, cultural, de grupo, de la temperatura, deben ampliarse las perspectivas. Y, propender a través de las escuelas de Arquitectura, de Artes y oficios, de Ingeniería, al desarrollo de la generación de energía barata y a la aislación térmica para lograr espacios habitacionales dignos para una vida digna. 
IV

Cuarta IDEA: Necesidad de subrayar l.A IMPORTancia

DEL HOMBRE TANTO COMC CONSTRU(:TOR QUE COMO POTENCIAL.

TRANSFORMADOR DEL AMBIENTE URBANO

Debe subrayarse que es el hombre el que ha construido el medio ambiente urbano total. Es decir, todos estos pisos de la construcción social: el piso biológico, el cultural, el psicológico o del alma, el de las relaciones sociales o de los grupos. Y, aun, gran parte del ambiente natural incorporado a la urbe.

Asimismo, la educación debe mostrar que, así como el hombre los ha construido y experimenta los beneficios de esta construcción, también el hombre experimenta los problemas, a veces trágicos, que le traen sus propios diseños ambientales, cuando son deficientes.

Es importante que la educación muestre a los vecinos y a los niños que son los candidatos a futuros vecinos, con casos concretos, simples en un comienzo, más complejos en niveles superiores de la educación, que el hombre es capaz de entender, estudiar, describir el medio social, desmontar las partes que constituyen esta compleja máquina. Pero, lo que es todavía más importante, que el hombre puede intervenir, recomponer, reconstruir, rediseñar tal ambiente. Que es capaz de prevenir, de hacer una educación preventiva y, como dicen los prospectivistas o cientistas del futuro, que es capaz de formular una educación urbana prospectiva.

Estos ejemplos tienen que llegar a ser, en definitiva, más complejos que la simple demostración de cómo se diseña un puente. Porque deben mostrar que el hombre al cambiar el medio físico, con su diseño, produce voluntariamente o, tal vez, si preverlo, cambios en las relaciones sociales, la vida mental de las personas, de los grupos y de la biología. Deben los ejemplos mostrar que también profesionales como los arquitectos, los cientistas de la ciudad, cuando la intervienen, deben estar conscientes no sólo de la anatomía y la fisiología visible, o lo que podríamos llamar el fenoripo de la ciudad. También deben considerar su Genotipo, es decir, estas estructuras suplementarias, intersticiales, invisibles, que en sus combinaciones producen un sustancial metabolismo urbano, saludable o patológico.

Pero las ideas anteriores sobre la posibilidad de cambiar el medio, pueden ser demasiado teóricas y especulativas. Por eso quisiera ilustrar una de estas actividades de cambio, apoyándome en la investigación que realizamos hace años con el arquitecto, profesor don Fernando Larraguibel, en la calle Juan Vicuña en Santiago*. Hoy mismo, por lo menos, las manifestaciones materiales de esa

* El estudio en referencia forma parte del proyecto Diseño y Organización Experimental en el Medio Ambiente Vecinal, cuyo investigador responsable fue el profesor Fernando Larraguibel, y en el cual el autor de este artículo participó como investigador desde su iniciación (proyecto $\mathrm{H}-449824 \mathrm{~F}-1980$, proyecto patrocinado por la Oficina de Desarrollo Científico de la Universidad de Chile, hoy Departamento de Investigación y Bibliotecas de la misma Universidad). Esta experiencia chilena ha sido comentada en U.S.A., por Westland y Knight, 1982. 
experiencia pueden ser observadas, todavía, por cualquier vecino. En unas fotografías de esta calle pueden apreciarse algunos aspectos de esta experiencia de cambio (ver Fotos 3, 4, 5 y 6 ).

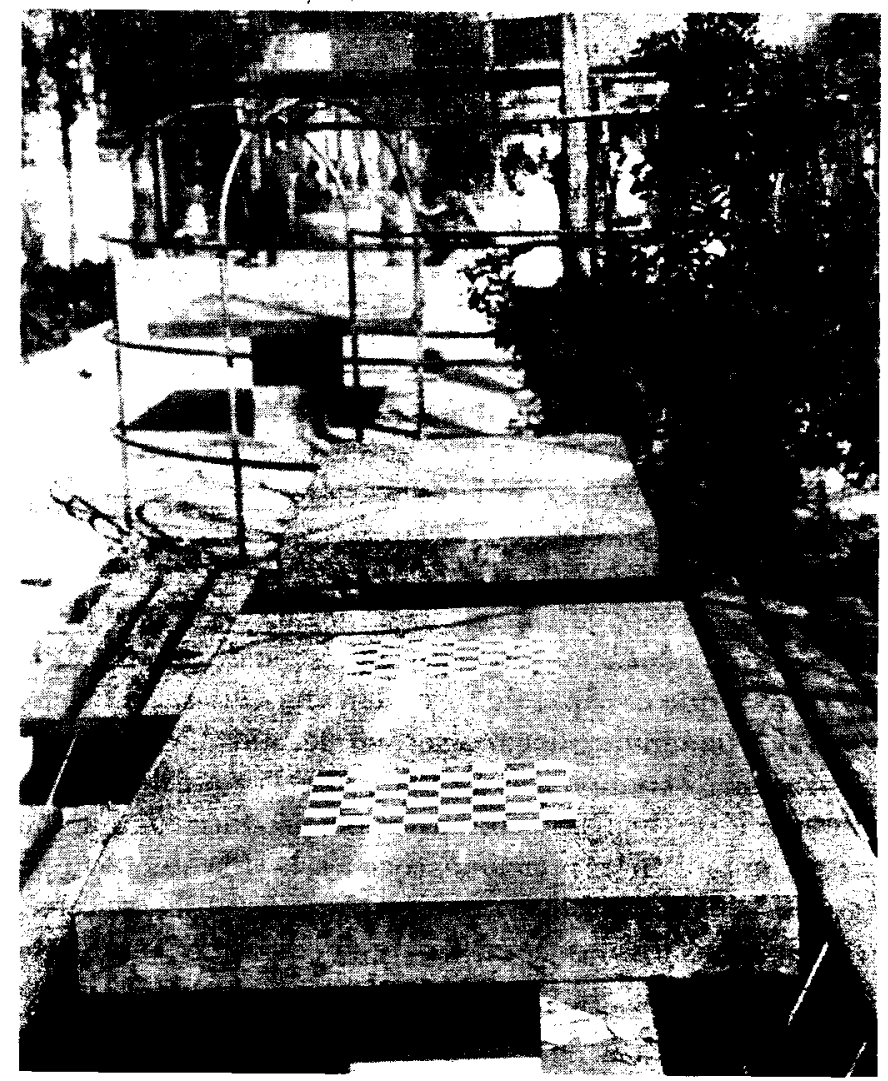

Foto 3. En primer plano, mesas para ajedrez. que tienen también otros usos: almorzar. estudiar. etc. En segundo plano. detalle de la "ducha-juego", Los niños juegan ! pasan bajo la ducha, consiguiendo, espontáneamente, la limpieza de sus cuerpos por chorros que surgen de las cañerías (Foto de Julio Munizaga F., julio, 1985).

Eramos, en ese tiempo, un equipo de profesionales, presidido por el Arquitecto, Prof. Fernando Larraguibel, entre los que estaban el Dr. Enrique Fanta, el sociólogo Julio Munizaga, la educadora Ana Downey. Teníamos la incalculable contribución de la Dirección de Deportes del Estado, de la Ilustre Municipalidad de Santiago y de la Oficina de Desarrollo Científico (hoy D.I.B.) de la Universidad de Chile. Fue decisiva la entusiasta e idónea cooperación y confianza que puso en nosotros el Prof. Carlos Aliaga, actual Director del Museo de Santiago, y del Departamento de Obras Municipales de la Municipalidad de esta capital. 
(ILDAD Y VECINOS: CUATRO IDEAS PARA LA ENSENANZA I)E I.A VII)A LRBANA

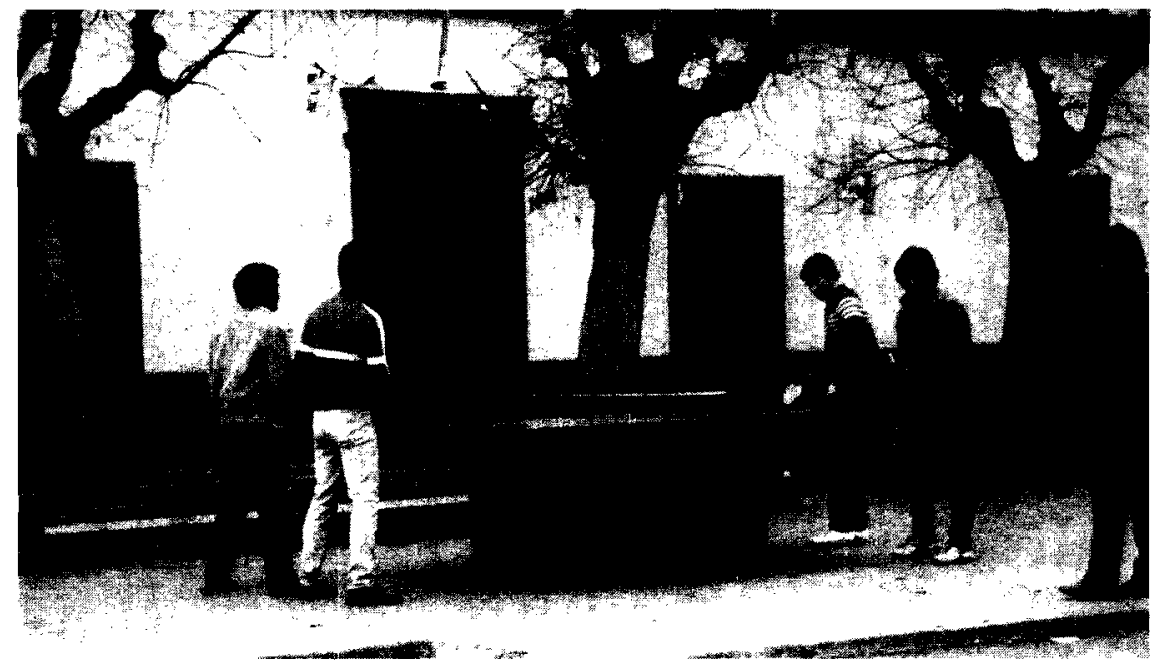

Foto 4. En la calle las mesas de ping-pong se siguen usando por los vecinos (Folo de Julio) Munizaga F., julio, 1985).

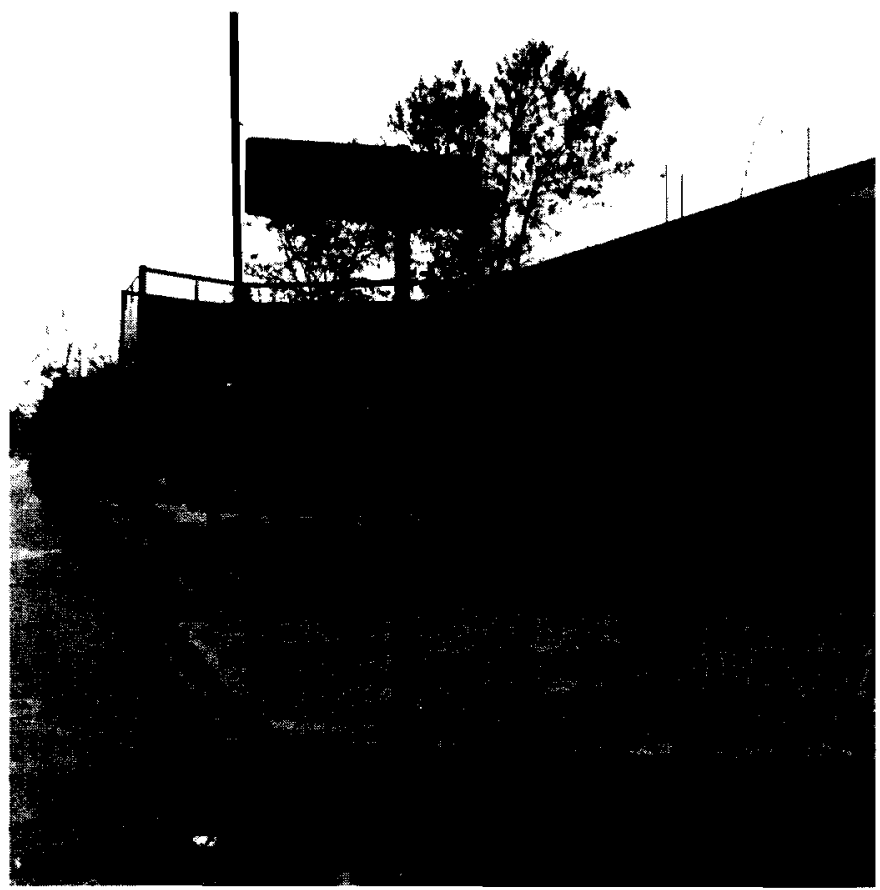

Foto 5. Los vecinos, especialmente nin̄os y adolescentes, siguen subiendo al puente o "mirador" que atraviesa la vereda (Detalle del puente y su uso en la Foto 6) (Foto de Julio Munizaga F., julio. 1985). 


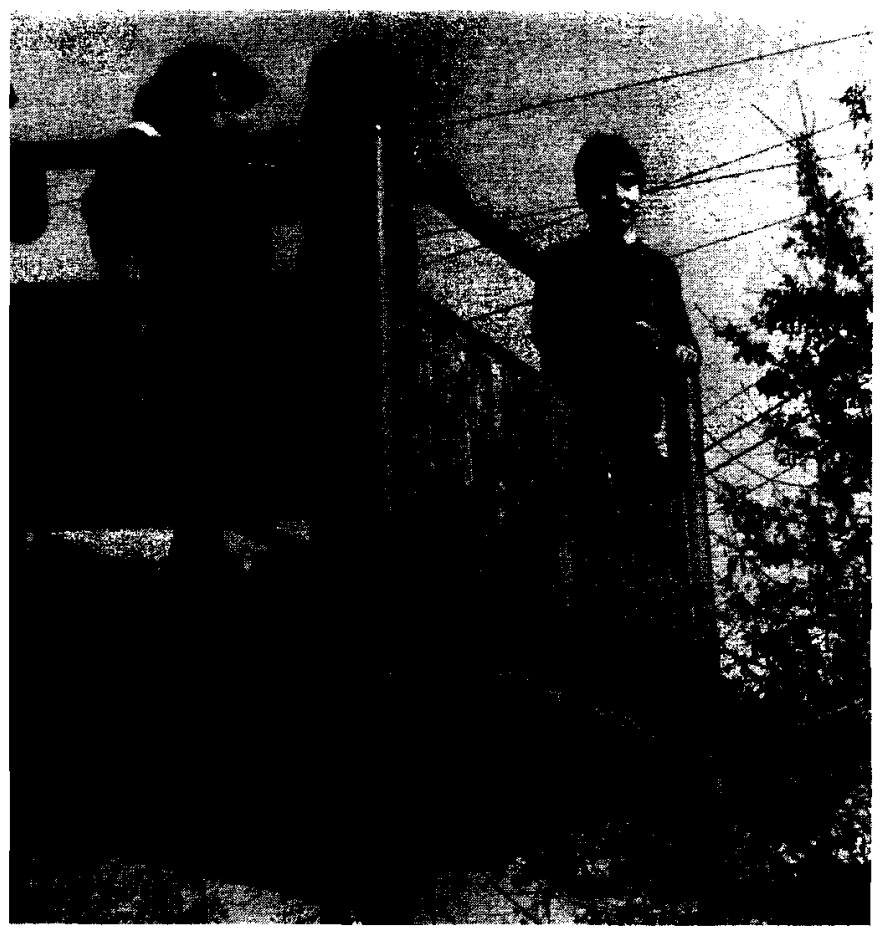

Folo 6 (Detalle del puente o mirador). Niños sobre el mirador. Ellos lo utilizan para observar el paisaje urbano y medilar. El adolescente del extremo derecho dijo usarko para este último fin. (Foro de Julio Munizaga F., julio, 1985).

Se trata de una pequeña calle ubicada al Sur de Av. Matta. En esta corta calle, de gente de pocos ingresos, de casas de un piso, había poca interacción de los vecinos. Nos preocupaba mucho la enorme cantidad de niños que pasaba su vida viviendo encarcelados en el interior de sus casas y en los cités. Las funciones de esta calle eran fundamentalmente peatonales, los vecinos salían al trabajo y llegaban para desaparecer en el interior de sus habitaciones. La calle era solitaria, sin vida social y los automóviles pasaban con gran velocidad, poniendo en peligro la vida de los pequeños.

Nos propusimos cambiar las funciones de esta calle, convertirla en sitio para incrementar la vida social. Para este efecto se modificó la estructura tradicional de la calle en calzadas y veredas. Buscamos estimular las mentes y el sentido estético de los pequeños, dándole decoración y dibujos nuevos al piso de la calle. Consultando la opinión de los vecinos, las fachadas de las casas fueron pintadas por ellos mismos, con los colores que percibían como agradables. Además, los vecinos recibieron clases teóricas y prácticas de apreciación estética, con la colaboración de profesores de Arte y Educación de la Universidad de Chile. El entonces Decano de la Facultad de Arte Prof. Kurt Herdan guió estos aspectos. 
La calle tiene hoy un aspecto muy especial porque cada vecino pintó las fachadas de colores no tradicionales. Cada grupo de vecinos construyó jardines frente a su casa; domesticó este espacio que es baldío en la mayor parte de las calles de Santiago. Se modificó también la calzada y una parte de ella se dedicó a canchas y pistas para que pudieran realizarse funciones deportivas y de recreación. Se construyeron desniveles y cercos para controlar el tránsito, por momentos, durante la recreación y el deporte. En principio, se diseñaron sectores específicos para el uso de las personas de más edad, para los adolescentes, para los pequeños y los párvulos. Y un espacio para la familia y la comunidad. Se ubicaron mesas fijas para que la gente practicara ajedrez y otros juegos, se reunieran, conversaran, trabajaran y estudiaran. $Y$ recordando esa percepción "enemiga" que habia del sector Sur con el sector Norte de la calle, construimos, justamente en el centro, un escenario y una multicancha que sirviera de lugar integrador para actos cívicos, públicos, recreativos de los vecinos. La Ilustre Municipalidad de Santiago dictó un Decreto para regular una menor velocidad de los vehículos y también se reforzó la iluminación eléctrica para iluminar las actividades deportivas y de recreación. Se instaló una especie de gran puente al que podía subirse, pensando con esto darles a los niños y a los vecinos la posibilidad que, desde pequeños, pudieran percibir el espacio desde la altura.

El cambio de esta calle constituye un verdadero laboratorio de investigación, una ventana que permite percibir aspectos ocultos e intersticiales cuya ignorancia imposibilita comprender la vida en la pequeña escala y, entonces, dificulta la preparación del vecino para participar en escalas urbanas humanas sucesivamente mayores.

Podemos decir que en este experimento social-urbano se produjeron cambios en la interacción de los individuos, ya que en la tarea misma de modelación participaron todos, adultos y niños. Se vio a los padres y a los ancianos jugar con sus hijos y cuidar a los nietos en los juegos colectivos. Vino gente de barrios lejanos a observar este cambio. Las Organizaciones vecinales actuaron y, como es humano, ocurrieron los conflictos que tenían que ocurrir.

Pero quien no haya estudiado la teoría social del conflicto y no sepa que éste tiene también resultantes positivas, nunca podrá evaluar los frutos de las discusiones, de los distanciamientos, de las pugnas, de los incidentes que allí ocurrieron.

Las madres, con tranquilidad, dejaron que sus pequeños hijos, de dos o más años, jugaran confiados en el sector asignado y educadoras de párvulos hicieron su práctica, enseñándoles en la calle. Por eso, hasta hoy, el experimento se conoce como el de la Calle-Escuela. Los monitores de la Dirección General de Deportes y del Comité Nacional de Recreación entrenaron a los niños. Todos éstos fueron actos públicos que presenciaban viejos, jóvenes y niños. En uno de los experimentos en la calle se les entregó a diversos grupos de edad, incluyendo niños bien pequeños, la tarea de organizar, vigilar y preservar los sectores que correspondían a sus edades. Y, en el escenario del punto central de la calle, se vio concurrir 
a los vecinos antagónicos de los sectores Sur y Norte de la calle, sin que pensemos, ingenuamente, que esto constituye una formidable integración.

No diré más de este ejemplo que está allí a la vista de todos, que la Ilustre Municipalidad de Santiago sigue protegiendo. Sólo terminaré recordando que se dice que en Chile no producimos muchas ideas exportables; que siempre importamos lo externo. Curiosamente, esta tarea nuestra de la calle Juan Vicuña, poco conocida en Chile, es bastante conocida en el extranjero. Unos arquitectos urbanistas argentinos que vinieron expresamente a estudiar la calle, quisieron tomar fotografías y planos. Pero los vecinos se lo impidieron. La gente de la calle nos explicó que no querían que esta construcción a la que parecían asignarle caracteres extraordinarios, de difícil imitación y de la que se sentían orgullosos, fuera copiada por extranjeros. Pero, esta experiencia fue utilizada por expertos urbanistas en Israel. La información personal del Prof. Hernán Emeres Y., Gerente General de la Asociación Cristiana de Jóvenes de Chile, es la siguiente: en 1980, el Sr. Presidente de la Asociación 1sraelita de Recreación Hillel Ruskin y la Universidad de Jerusalem, pidieron los antecedentes del Proyecto "Calle Escuela" para realizar una experiencia piloto, semejante a la chilena, en Jerusalem, con los Centros Comunitarios. Ellos han citado nuestra experiencia que para ellos era novedosa y que, al mismo tiempo, les permitía lograr propósitos importantes para su ciudad. Especialmente, lograr cambios urbanos que permitieran la integración de los vecinos. Porque en Israel existe el problema de integrar a gentes de culturas muy diferentes que han migrado a la ciudad: pakistanos, hindúes, árabes, africanos. Además, nuestra experiencia aparece citada como un experimento social por Westland y Knight, 1982 (pp. 145-146).

En fin, en 1srael se interesaron por el cambio del ambiente urbano de una calle. Justamente, como nosotros aquí en Santiago, con el objeto de lograr un cambio en la organización social, en la participación, en la integración, ell la personalidad, en la calidad del tejido oculto e intersticial de la vida urbana. Repito que éste sería un ejemplo que debería elaborarse en las escuelas de Santiago y provincias; en el Liceo y en la Universidad y compararlo con la experiencia de lsrael y otras de diferentes países. Serviría para ilustrar, con ejemplos cercanos, chilenos, esta capacidad del hombre para cambiar el medio urbano en relación con necesidades críticas que requiere el desarrollo integral digno de los vecinos. Se trata de un ejemplo en que el cambio lo producen "profesionales" y cientistas. Pero, nuestra obligación y la de los pedagogos, es mostrar que esto no lo hacen sólo los expertos. Todos los vecinos están constantemente, sin saberlo, transformando la ciudad material y la ciudad moral. Y la escuela debe ser capaz de señalar y orientar la potencialidad y el sentido de este proceso.*

* El autor realizó una visita a la calle en julio de 1985 , y a raiz de ella conviene dejar constanciá que se constatan deterioros en el equipamiento material, algunos por causa del tiempo y otros, según los vecinos, por problemas de los lideres a cargo. Se requiere, pues, un reestudio de todo el provecto. 
Estimamos que las cuatro ideas que hemos expuesto pueden incorporarse en un núcleo para elaborar una pedagogía urbana. Cada idea constituye una especie de orientación de valor, cuyo contenido concreto puede variar, adaptarse, según las regiones del pais, los grupos humanos, el clima, el contexto sociocultural y económico.

Es deseable que estas cuatro orientaciones produzcan cambios en la conducta social urbana real y no sólo cambios psicológicos individuales y de actitudes. Esto depende de la metodología pedagógica y del contexto social total dentro del que se produzca la enseñanza.

\section{Bibliografia}

Banton, Michel; Wolf, Eric y otros (comp.). Antropologia Social de las Sociedades Complejas. Alianza Editorial, Madrid, 1980.

Inkeles, Alex y Smith, David. Becaming Modern. Harvard University Press, 1974.

Munizaga, Carlos. Reconocimienlo Cultural de Chiloé. Departamento de Ciencias Antropológicas y Arqueológicas. Facultad de Filosofía y Educación. Publicación $\mathrm{N}^{\circ} 20,1971$.

Mi nizacia, Carlos. "Notas sobre adaptación al Medio Ambiente Físico de ('hiloé, Vivienda y Urbanismo". Departamento de Ciencias Antropológicas y Arqueológicas. Guía de Trabajo de Antropología Social U. de Chile, 197I b.

Munizacia, Carios. Reconocimiento Cullural de Chiloé. Isla Grande. Aspectos Antropológicos (inéd.). Universidad de Chile, 1978.

Mlinizadia, Carlos. Arqueologia: Algunas Funciones L'rbanas y de Eduración, Anlecedentes para el Estudio de "Sitios Testigo" en Santiago de Chile. Revista Codeci [Santiago]. N" I. Fascículo 2, septiembre, 1981.

Munizacia, Carlos. Marco para un Enfoque sobre Municipio y Salud. Revista de Ciencias Sociales, publicación de la Facultad de Ciencias Jurídicas, Económicas y Sociales. Universidad de Valparaíso. [Valparaíso] $\mathrm{N}^{\circ} 24$, $1^{\mathrm{el}}$ semestre, pp. 187-205.

Toffler, Alvin. La Terceta Ola. Plaza y Janés Editores. Barcelona, 1980.

Westland, Cor; Knight, Jane. Playing Living Learning. A Worldwide Perspective on Children Opportunities to Play. Venture Publishing Inc. State College, usA., 1982. 